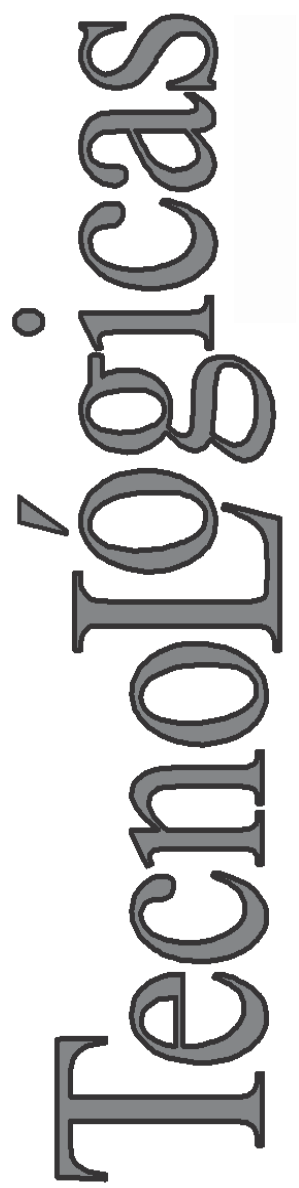

\title{
Simulación Numérica de la Combustión con Aire Enriquecido en un Horno de Fusión de Fritas
}

\section{Numeric Simulation of Oxygen Enriched Combustion in a Frit Melting Kiln}

Bernardo A. Herrera-Múnera ${ }^{1}$ Luis H. Copete-López ${ }^{2}$ Jhony M. Gutiérrez-Flórez ${ }^{3}$ Raúl A. Ortega-López ${ }^{4}$

1 Facultad de Ingeniería, Instituto Tecnológico Metropolitano, Medellín-Colombia bernardoherrera@itm.edu.co

2 Soluciones Energéticas y de Automatización, Medellín-Colombia gerencia@iosoluciones.co

3 Soluciones Energéticas y de Automatización, Medellín-Colombia ingenieria@iosoluciones.co 4 Departamento de Soporte de Procesos, Suministros de Colombia, Medellín-Colombia rortega@corona.com.co 


\section{Resumen}

En este trabajo se presenta un estudio numérico de la combustión con aire enriquecido de un horno rotatorio para la fundición de frita que usa gas natural como combustible, con el fin de determinar una concentración de oxígeno que garantice la viabilidad económica del proceso sin afectar los requerimientos específicos de calidad. Las simulaciones se realizaron utilizando el software comercial ANSYS FLUENT como herramienta de diseño para predecir el comportamiento del sistema térmico y establecer puntos de funcionamiento con diferentes niveles de enriquecimiento del aire. Se empleó el modelo Finite Rate / Eddy Dissipation para la combustión, mientras que los modelos $\mathrm{k}-\varepsilon$ Realizable y Ordenadas Discretas fueron usados para la simulación del flujo turbulento y la radiación, respectivamente. Los resultados mostraron que un nivel de enriquecimiento cercano al $31 \%$ de oxígeno en el aire permite alcanzar las temperaturas de fusión de la frita mayores a $1700 \mathrm{~K}$, logrando así una disminución en los consumos actuales de oxígeno de alta pureza sin afectar la producción y calidad del producto.

\section{Palabras clave}

Fundición de frita; combustión con aire enriquecido; dinámica de fluidos computacional.

\section{Abstract}

In this paper, a numerical study of air enriched combustion on a natural gas rotary furnace for frita melting is presented. This study was done with the aim of determining an oxygen concentration to ensure economic feasibility of the process without affecting quality requirements. The simulations were conducted using the commercial software ANSYS FLUENT as a design tool to predict the behavior of the thermal system and to establish operations conditions with different oxygen enrichment levels. Finite Rate / Eddy Dissipation model was used for combustion simulation, while $\mathrm{k}-\varepsilon$ Realizable and Discrete Ordinates models were utilized for turbulence and radiation simulation, respectively. It was found that an enrichment level close to $31 \%$ of oxygen in the air allows for reaching temperatures for frita melting larger than $1700 \mathrm{~K}$. In this way, current consumption of high purity oxygen can be diminished without affecting the production levels and the quality of the product.

\section{Keywords}

Frit melting; oxygen enriched air combustion; computational fluid dynamics. 


\section{INTRODUCCIÓN}

El proceso de producción de fritas tiene como objetivo la obtención de un material vítreo insoluble en agua a través de la fusión y posterior enfriamiento de una mezcla de diferentes materias primas de naturaleza cristalina. El proceso industrial se realiza a temperaturas elevadas comprendidas entre $1400{ }^{\circ} \mathrm{C}$ y $1600{ }^{\circ} \mathrm{C}$ dependiendo del tipo de frita que se desee producir. Una masa fundida se forma en el proceso para ser sometida a un enfriamiento brusco en agua con el fin de mantener su estructura vítrea y favorecer su disgregación en pequeños gránulos o escamas de vidrio.

El proceso de fusión generalmente se realiza en hornos rotatorios o continuos que por las elevadas temperaturas requieren la utilización de oxígeno puro como comburente, incrementando los costos del proceso productivo. El combustible y el oxígeno son suministrados a quemadores generalmente de llama de difusión para reaccionar entre ellos en una gran cámara liberando una considerable cantidad de calor en forma de radiación y convección.

En las simulaciones numéricas de este proceso, las reacciones de combustión, la turbulencia de los gases, la cinética química, el proceso de fundición, las burbujas de gas, las partículas sólidas y los fenómenos térmicos y fluidodinámicos son los principales parámetros de estudio. Falcitelli et al. (2002) presentaron una metodología para la simulación de sistemas prácticos de combustión y la predicción de NOx sobre dos hornos de fundición de vidrio. La evaluación de los modelos empleados se aproximó adecuadamente a las mediciones de perfiles de temperatura y la predicción de NOx.s.

Chang et al. (2005) realizaron un modelo computacional usando la aproximación Euleriana para simular flujos multifásicos en un tanque de fusión de vidrio, incluyendo todas las fases, dividiendo partículas sólidas o burbujas gaseosas en grupos de varios tamaños y tratando cada grupo como medio continuo.

Por su parte, Abbassi y Khoshmanesh (2008) realizaron un estudio tridimensional de un horno de fusión de vidrio regenerativo, en el cual las temperaturas calculadas y medidas en diferentes zonas indicaron un máximo error relativo de $7.7 \%$ con un método 
que desacopla la zona de combustión con la zona de fusión, haciéndose más flexible y rápido que los métodos convencionales.

Ponsich et al. (2009) realizaron el modelado de un horno de fusión de vidrio con software CFD, desacoplando la zona de combustión y de fusión. Cada zona fue descompuesta en una red de reactores que reproduce la hidrodinámica del medio. Más adelante, Possamai et al (2012) simularon un horno de fusión de fritas de gas natural y oxígeno puro como comburente mediante el software CFX. Los resultados mostraron una predicción muy aproximada de la tendencia de la temperatura de la pared externa, identificando puntos calientes que deben evitarse para la operación óptima del horno. Así mismo, los autores realizaron una simulación para optimizar la geometría y el material del aislamiento con el fin de disminuir las pérdidas de calor.

Teniendo en cuenta que al sustituir el consumo de oxígeno puro por medio del uso de corrientes de aire con una concentración de oxigeno superior a 21\% (aire atmosférico) se pueden reducir los costos de producción, en este trabajo se busca determinar por medio de la simulación numérica los niveles de enriquecimiento de aire, manteniendo constantes el consumo de gas natural y el factor de aireación en el sistema, para las cuales no se afecten las temperaturas de operación en el proceso de fusión de frita en un horno rotatorio. Algunos resultados numéricos fueron validados por medio de mediciones de temperatura en 5 puntos de la superficie interior de la pared del horno y mediciones de NOx.

\section{METODOLOGíA}

\subsection{Descripción de la Geometría del Horno de Fusión de Frita}

Se reprodujo la geometría del horno rotatorio $\mathrm{N}^{\circ} 2$ de la planta Colorificio de Sumicol - Corona, el cual es presentado en la Fig. 1. Tiene un volumen total de $1.22 \mathrm{~m} 3$, de los cuales $0.188 \mathrm{~m} 3$ son ocupados por el material vítreo a fundir. La potencia térmica del sistema es $600 \mathrm{~kW}$, la temperatura de fusión de la frita según su referencia oscila entre $1400^{\circ} \mathrm{C}$ y $1600^{\circ} \mathrm{C}$, temperaturas que son 
difíciles de alcanzar con aire normal lo que llevó a la implementación de la combustión con oxígeno puro al 99.9\% de pureza.

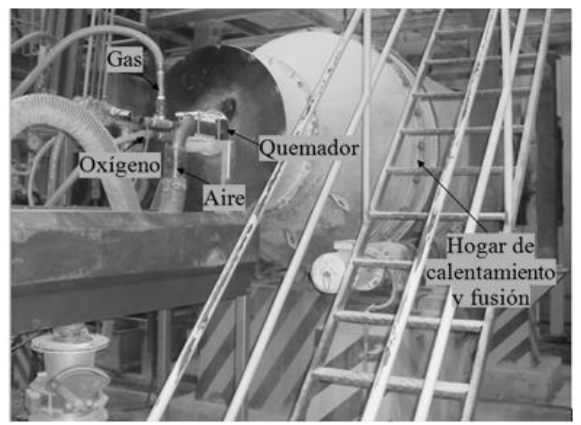

Fig. 1. Horno rotatorio de fusión de frita. Fuente: Autores

\subsection{Simulación Numérica}

Para el estudio de este sistema turbulento y reactivo se emplearon las ecuaciones de conservación de continuidad, cantidad de movimiento energía y especies químicas de la forma promediada de Favre que involucra los efectos debido al cambio de densidad. Para una comprensión detallada de la deducción e interpretación física de las ecuaciones de conservación se recomienda la revisión de trabajos de Patankar (1980) y Verstegg y Malalasakera (1995).

\subsubsection{Modelos fenomenológicos}

Como modelo de combustión e interacción entre química y turbulencia se usó en la simulación el Finite Rate / Eddy Dissipation propuesto por Magnussen y Hjertager (1976) con un mecanismo de combustión del gas natural de dos pasos propuesto por Westbrook y Dryer (1981).

Para la simulación de la turbulencia se seleccionó el modelo k$\varepsilon$ realizable con las constantes por defecto $\left(\mathrm{C}_{1 \varepsilon}=1.44, \mathrm{C}_{2}=1.9, \sigma_{\mathrm{k}}=\right.$ $1.0, \sigma_{\varepsilon}=1.2 \mathrm{y} \mathrm{C}_{\mu}=0.09$ ) propuesto por Shi et al (1995) debido a que ofrece una mejor predicción de la tasa de esparcimiento generada por chorros redondos. Por otra parte, se seleccionó el modelo Ordenadas Discretas (Raithby \& Chui, 1990; Chui \& Raitby, 1993) para la transferencia de calor por radiación desde las paredes y 
desde los gases de combustión hacia la carga a fundir y se incorporó el modelo de Suma Ponderada de Gases Grises (Smith et al, 1982; Coppalle \& Vervisch, 1983; Denison \& Webb, 1993) para considerar la influencia del vapor de agua y el CO2 sobre los patrones de radiación. Para la simulación de la formación de NOx se utilizaron los dos mecanismos principales de formación de $\mathrm{NOx}$ en la combustión de metano aire, un mecanismo súbito (Fenimore, 1971) y un mecanismo térmico (Miller \& Bowman, 1989).

\subsubsection{Método de solución}

Para resolver los modelos matemáticos, el software ANSYS FLUENT convierte las ecuaciones diferenciales del modelo en ecuaciones algebraicas por medio del método de volúmenes finitos. El sistema de ecuaciones se resolvió usando el método segregado y la linealización de las ecuaciones algebraicas se realizó por medio de un esquema implícito (Veersteg \& Malalasakera, 1995).

El dominio computacional para la simulación CFD incluye un quemador de llama de difusión, un tramo divergente, el hogar de fusión cilíndrico y otro tramo convergente hasta llegar a la evacuación de los gases como puede apreciarse en la Fig. 2. Sólo se simuló la mitad longitudinal del horno se impuso una condición de frontera de simetría con el fin de reducir el costo computacional de la simulación. El mallado interno del sistema cuenta con un total de 1.290 .843 celdas tetraédricas, una oblicuidad máxima de 0.84 y una relación de aspecto máxima de 12 .

\subsubsection{Condiciones de simulación}

La metodología para la simulación partió de definir propiedades de entrada como potencia térmica, carga de frita y composiciones del comburente. Luego de realizar simulaciones previas, se encontró la composición del comburente como la variable más significativa en el proceso de fusión y se establecieron 4 niveles de enriquecimiento de aire con $21 \%$ (aire ambiente), 31\%, 50\% y $100 \%$ (oxicombustión) de volumen de oxígeno en el comburente. Simultáneamente se solucionaban las ecuaciones de continuidad, momentum, energía, especies químicas, turbulencia, radiación. En las Tablas 1 y 2 se presentan las condiciones de frontera para cada una de las simulaciones realizadas y las propiedades de la frita, respectivamente. 
Tabla 1. Condiciones de frontera para cada simulación. Fuente: Autores

\begin{tabular}{lcccc}
\hline \multirow{2}{*}{ Propiedad } & \multicolumn{4}{c}{ \% de O2 en el aire } \\
\cline { 2 - 5 } & 21 & 30 & 50 & 100 \\
\hline Potencia $(\mathrm{kW})$ & \multicolumn{4}{c}{600} \\
Flujo de gas natural $(\mathrm{kg} / \mathrm{s})$ & 0,0126 \\
Flujo de aire enriquecido $(\mathrm{kg} / \mathrm{s})$ & 0,292 & 0,145 & 0,089 & 0,068 \\
Flujo de frita $(\mathrm{kg} / \mathrm{s})$ & \multicolumn{4}{c}{1,5} \\
\hline
\end{tabular}

Para la validación experimental, se realizaron 3 fusiones con un enriquecimiento de $31 \%$ de $\mathrm{O} 2$ en el aire. En cada fusión se midió la temperatura de pared interna del horno por medio de un pirómetro infrarrojo, ajustando en cada caso la emisividad del aislamiento en el interior y el acero en la carcasa exterior. En el área de salida del horno se midió la concentración de NOx con un analizador de gases que opera bajo el principio de infrarrojo no dispersivo.

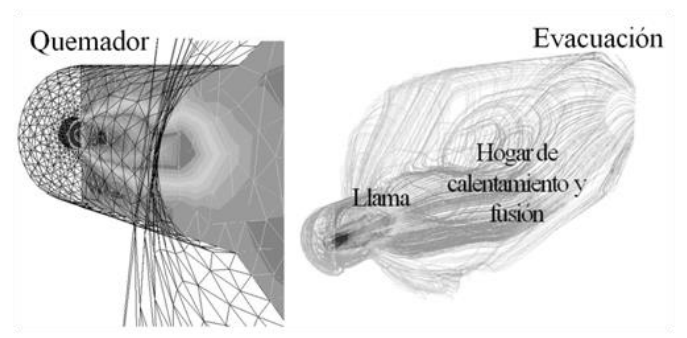

Fig. 2. Dominio computacional. Fuente: Autores

Tabla 2. Propiedades de la frita fundida. Fuente: Autores

\begin{tabular}{lcc}
\hline \multicolumn{1}{c}{ Propiedad } & Unidad & Valor \\
\hline Densidad & $\mathrm{kg} / \mathrm{m}^{3}$ & 2203 \\
Calor específico & $\mathrm{J} / \mathrm{kg} \cdot \mathrm{K}$ & 733 \\
Conductividad térmica & $\mathrm{W} / \mathrm{m} . \mathrm{K}$ & 1.3 \\
Viscosidad & $\mathrm{kg} / \mathrm{m} . \mathrm{s}$ & 2948 \\
\hline
\end{tabular}




\section{RESULTADOS Y DISCUSIÓN}

Los perfiles de temperatura de la Fig. 3 muestran que a partir de porcentajes de enriquecimiento de $31 \%$ de oxígeno en el comburente, el sistema es adecuado para alcanzar temperaturas de pared superiores a $1500{ }^{\circ} \mathrm{C}$, temperatura necesaria para llevar a cabo el proceso de fusión en forma eficiente. Las simulaciones muestran que con aire del ambiente no es posible alcanzar temperaturas mayores a $1400{ }^{\circ} \mathrm{C}$ debido a la gran cantidad de gases inertes resultantes de la combustión.

Se eligió un enriquecimiento con $31 \%$ de oxígeno en el aire como punto de operación de referencia para la medición experimental, ya que este punto implica el menor consumo de oxígeno industrial para el enriquecimiento. Las mediciones de temperatura de pared interna muestran una alta correspondencia con las respectivas temperaturas obtenidas en la simulación numérica (Fig. 3), especialmente en la segunda mitad del horno, lo cual valida el software CFD utilizado como herramienta de análisis y diseño adecuada para el estudio de mejoramiento del horno rotatorio. En este caso, la mayor desviación relativa entre los datos experimentales y numéricos fue del $4.4 \%$.

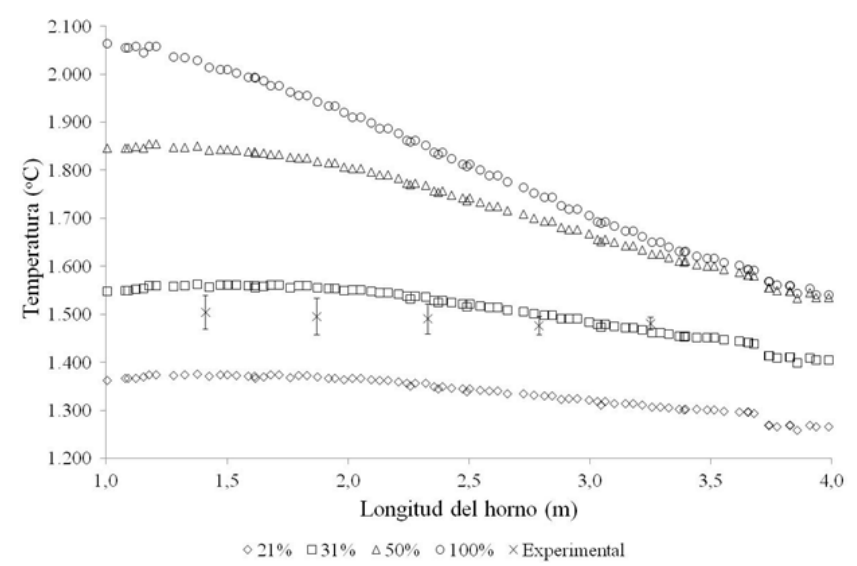

Fig. 3. Perfiles de temperatura en la pared interna del horno rotatorio. Fuente: Autores 
Cabe notar como con porcentajes de enriquecimiento de 50\% y oxicombustión con oxígeno puro, las temperaturas en la pared interna pueden llegar a valores superiores a $1800{ }^{\circ} \mathrm{C}$ e incluso de $2000{ }^{\circ} \mathrm{C}$, con lo cual se corre el riesgo de sobrecalentar el material y de afectar significativamente la durabilidad de los componentes del horno, además que implica un mayor consumo de oxígeno y por ende un aumento en los costos de producción. Esta es la razón por la cual el control de temperatura en los hornos de fusión de frita se convierte en un parámetro de vital importancia para garantizar la calidad del producto.

Los perfiles de temperatura de la Fig. 4 muestran lo diferente que resulta el fenómeno de la combustión en la zona de reacción cercana al quemador cuando se aumenta la concentración de oxígeno en el comburente. La combustión con aire del ambiente resulta en un perfil plano a partir de 1 metro desde el quemador, pero en ningún momento la temperatura sobrepasa los $1500{ }^{\circ} \mathrm{C}$, condición necesaria para realizar la fusión del material vítreo. Al tener la condición experimental de $31 \%$ de oxígeno en el comburente, el perfil de temperatura se asemeja mucho al perfil con aire del ambiente por ser muy uniforme desde aproximadamente $70 \mathrm{~cm}$ desde el quemador, pero alcanzando en todo el recorrido temperaturas mayores a $1500{ }^{\circ} \mathrm{C}$. Este perfil resulta beneficioso para la calidad del producto puesto que proporciona un calentamiento uniforme a lo largo del horno y evita un sobrecalentamiento innecesario al inicio del recorrido del material. Esto último ocurre con el enriquecimiento de aire al $50 \%$ de oxígeno y con la oxicombustión, las cuales muestran altos picos de temperatura en la sección inicial del horno, alcanzando 2800 y $3600{ }^{\circ} \mathrm{C}$, respectivamente.

$\mathrm{Si}$ bien la alta temperatura favorece una mayor transferencia de calor por radiación hacia la carga, también debe tenerse en cuenta que esta puede afectar los componentes del quemador y del horno, aumentando los costos de mantenimiento. Con base en lo anterior, se puede concluir que la elección de un enriquecimiento de $30 \%$ para la operación del horno es acertada desde el punto de vista de la obtención de temperaturas más uniformes y bajo consumo de oxígeno, sin afectar la calidad del producto. 


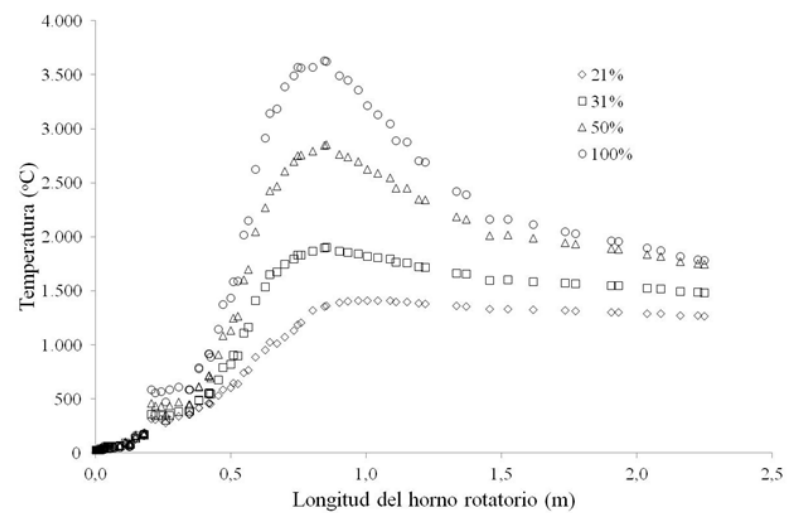

Fig. 4. Perfiles de temperatura en el eje longitudinal del horno rotatorio obtenidos mediante simulación numérica. Fuente: Autores

Por su parte, el análisis de NOx constituye un aspecto de primordial importancia en las aplicaciones de combustión con aire enriquecido con oxígeno, pues la formación de estas especies por medio del mecanismo térmico se hace significativa con las altas temperaturas que se alcanzan. Las mediciones experimentales del horno trabajando con $31 \%$ de oxígeno en el aire de combustión alcanzaron un valor de $275 \pm 40 \mathrm{ppm}$. La simulación numérica para las mismas condiciones arrojó un valor de $292 \mathrm{ppm}$, lo cual resulta ser una predicción adecuada de la formación de estas especies y que cumple con la legislación actual del país. Las emisiones de NOx más bajas obtenidas con simulación numérica fueron de 0.10 y $0.54 \mathrm{ppm}$ para oxicombustión y combustión con aire atmosférico, respectivamente, mientras que el valor más alto fue de 8322 ppm con $50 \%$ de oxígeno en el comburente, como consecuencia de la alta temperatura (superior a $2500^{\circ} \mathrm{C}$ ) y al contenido significativo de nitrógeno en el comburente. Estos resultados confirman que la elección de un enriquecimiento de aire hasta $30 \%$ de oxígeno no solo es beneficiosa desde el punto de vista de mejor control de la temperatura del proceso y bajo costo en el enriquecimiento, sino que también favorece una formación de NOx en niveles aceptables. 


\section{CONCLUSIONES}

Los resultados presentados en este artículo muestran las capacidades de los modelos empleados para la simulación tridimensional de un horno rotatorio de fusión de frita. La comparación de temperaturas de pared interna con mediciones experimentales indica que el máximo error relativo de los cálculos es menor al $5 \%$, lo que convierte a las simulaciones en una herramienta de gran utilidad para analizar el desempeño térmico del horno industrial. Sin embargo, la predicción de formación de especies contaminantes requiere el uso de modelos cinéticos más completos y conocer mejor la composición del combustible. No obstante, la simulación numérica predice bajos niveles de emisiones de especies como NOx, lo cual está en concordancia con los resultados experimentales y satisfacen la legislación vigente. Con ayuda de la simulación numérica se determinó que un enriquecimiento de 30\% de oxígeno en el aire de combustión produce valores de temperatura y especies contaminantes adecuadas para diferentes referencias de fritas comerciales para fabricación de esmaltes, sin incrementar significativamente los costos de producción.

\section{AGRADECIMIENTOS}

Este trabajo fue desarrollado dentro de la tesis de maestría titulada "Desarrollo de un banco de pruebas de combustión con aire enriquecido y oxi-gas para el incremento de la productividad en el proceso de fritas" del Instituto Tecnológico Metropolitano con el apoyo de la empresa SUMICOL S.A. a quien agradecemos su cooperación para la validación experimental de los resultados obtenidos.

\section{REFERENCIAS}

Abbassi, A., \& Khoshmanesh, K. (2008) Numerical simulation and experimental analysis of an industrial glass melting furnace. Applied Thermal Engineering, 28, 450-459. 
Chang, S., Zhou, C., \& Golchert, B. (2005). Eulerian approach for multiphase flow simulation in a glass melter. Applied Thermal Engineering, 25, 3083-3103.

Chui E., \& Raithby G. (1993). Computation of Radiant Heat Transfer on a Non-Orthogonal Mesh Using the Finite-Volume Method. Numerical Heat Transfer, Part B., 23, 269-288.

Coppalle A., \& Vervisch, P. (1983). The Total Emissivities of HighTemperature Flames. Combustion and Flame, 49, 101-108.

Denison, M., \& Webb, B. (1993). A Spectral Line-Based Weighted-Sum-ofGray-Gases Model for Arbitrary RTE Solvers. Journal of Heat Transfer, 115, 1002-1012.

Falcitelli, M.; Pasini, M., \& Tognotti, L. (2002). Modelling practical combustion systems and predicting NOx. Computers and Chemical Engineering, 26, 1171-1183.

Fenimore, C. (1971). Formation of Nitric Oxide in Premixed Hydrocarbon Flames. In 13th Symposium International on Combustion. The Combustion Institute, 373.

Launder, B. E., \& Spalding, D. B. (1972). Lectures in Mathematical Models of Turbulence. Academic Press, London, England.

Magnussen, B. F., \& Hjertager, B. H. (1976). On mathematical modelling of turbulent combustion with special emphasis on soot formation and combustion. The combustion institute, pp. 119-129.

Miller, J., \& Bowman, C. (1989). Mechanism and Modeling of Nitrogen Chemistry in Combustion. Progress in Energy and Combustion Science, 15, 287-338.

Patankar, S. V. (1980). Numerical heat transfer and fluid flow. $1^{a}$ ed., USA, Taylor \& Francis.

Ponsich, A., Azzaro-Pantel, C., Domenech, S., Pibouleau, L., \& Pigeonneau, F. (2009). A systemic approach for glass manufacturing process modeling. Chemical Engineering and Processing, 48, 1310-1320.

Possamai, T. S., Oba, R., \& Nicolau, V. P. (2012). Numerical and experimental thermal analysis of an industrial kiln used for frit production. Applied Thermal Engineering, 48, 414-425.

Raithby, G., \& Chui, E. (1990). A Finite-Volume Method for Predicting a Radiant Heat Transfer in Enclosures with Participating Media. Journal of Heat Transfer, 112, 415-423.

Shih, H., Liou, W., Shabbir, A., Yang, Z., \& Zhu, J. (1995). A New EddyViscosity Model for High Reynolds Number Turbulent Flows - Model Development and Validation. Computers Fluids, 24(3), 227-238.

Smith, T., Shen, Z., \& Friedman, J. N. (1982). Evaluation of Coefficients for the Weighted Sum of Gray ases Model. Journal of Heat Transfer, 104, 602-608. 
Versteeg, H. K., \& Malalasekera, W. (1995). An introdution to computational fluid dynamics. $1^{a}$ ed., New York, Longman Scientific \& Technical.

Westbrook, C., \& Dryer, F. (1981). Simplified reaction mechanisms for the oxidation of hydrocarbon fuels in flames. Combustion science and technology, 27, 31-43. 